\title{
GMO Detection Methodology
}

\author{
L. Grohmann \\ Federal Office for Consumer Protection and Food Safety (BVL), Berlin, Germany \\ Correspondence to: Dr. Lutz Grohmann, Federal Office for Consumer Protection and Food Safety (BVL), \\ Mauerstr. 39-42, D-10117 Berlin, Germany, Tel. +49 3018444 40510, E-mail: Lutz.Grohmann@bvl.bund.de
}

GMO detection is an essential part of the European regulatory framework concerning the environmental release of GMOs. While Directive 2001/18/EC is not strictly prescribing the detection methodology in case of an experimental release of GMOs in field trials, GMO authorisations for cultivation and placing on the market as food and feed according to Regulation (EC) No. 1829/2003 are rigidly coupled to particular requirements. For example, applicants have to deliver reference materials, methods for sampling, for matrix-specific DNA-extraction and for detection and event-specific quantification of the notified GMO.

Method requirements and detailed implementation guidelines for the scientific assessment and validation of the reference materials and the method protocols are defined by Regulation (EC) No. 641/2004. The Community Reference Laboratory (CRL) located at the IHCP/JRC in Ispra has to handle the core tasks with respect to the assessment of the GMO detection method, e. g. intra- and inter-laboratory validation according to specified method performance and acceptance criteria defined by the ENGL (European Network of GMO Laboratories). Furthermore, currently applied GMO methodologies are mainly based on the technical guidance and definitions given in Recommendation 2004/787/EC, e. g. use of DNA-based analytical strategies and the unit-of-measurement for GMO quantification (DNA copy number based).

Although a certain degree of harmonisation between current detection methodologies and legal requirements has been achieved in the EU, the presentation wants to address some of the challenging issues, e. g. correct identification and quantification of stacked events, efficient methodologies for GMO screening and problems arising from the low level presence of unauthorised events.

\section{Literature}

Directive 2001/18/EC of the European Parliament and of the Council on the deliberate release into the environment of genetically modified organisms and repealing Council Directive 90/220/ EEC. Official J Europ Comm 17.04.2001, p. No L106/1.

Recommendation 2004/787/EC. Commission Recommendation of 4 October 2004 on technical guidance for sampling and detection of genetically modified organisms and material produced from genetically modified organisms as or in products in the context of Regulation (EV) No 1830/2003.

Regulation (EC) No 1830/2003, traceability and labelling of genetically modified organisms and the traceability of food and feed products produced from genetically modified organisms and amending Directive 2001/18.

Regulation (EC) No. 641/2004. Commission Regulation (EC) No. 641/ 2004 on detailed rules for the implementation of Regulation (EC) No. 1829/2003 of the European Parliament and of the Council as regards the application for the authorisation of new genetically modified food and feed, the notification of existing products and adventitious or technically unavoidable presence of genetically modified material which has benefited from a favourable risk evaluation.

To access this journal online:

http://www.birkhauser.ch/JVL 